\title{
An Assessment of Community Finding Algorithms for Community-Based Message Routing in DTNs
}

\author{
Matthew Stabeler, Conrad Lee, and Pádraig Cunningham \\ School of Computer Science and Informatics \\ University College Dublin \\ Dublin 4, Ireland \\ matthew.stabeler@ucd.ie
}

\begin{abstract}
Previous work has demonstrated that community-finding algorithms can provide useful information for routing algorithms in delay tolerant networks. In this work we investigate which community finding algorithm most effectively informs this routing task. While early community finding algorithms partitioned networks into flat disjoint communities, more recent methods return community structures that can be overlapping and hierarchical. Given this diversity, it seems reasonable to expect that some methods will be better suited to message routing than others. In this paper, we evaluate a number of community finding strategies and find that Link Clustering, which returns overlapping hierarchical clusters, is very effective. We also find that InfoMap performs well - this is somewhat surprising given that InfoMap returns a flat partition of the network, however this may be because the optimization that drives InfoMap is based on flow.

Keywords: Delay Tolerant Networking, Community-Based Routing.
\end{abstract}

\section{Introduction}

The study of message routing in challenged networks $[18,5,11,10]$ has evolved to cover a variety of situations where traditional point to point networks may be hard, expensive, or otherwise infeasible to create or maintain. The term Delay Tolerant Networks (DTNs) covers many types of networks [2], such as InterVehicular communications, Mobile/Fixed Sensor networks (Animal, Human, Infrastructure etc.), satellite communications, battlefield communication networks, or any other system of interacting devices, objects, beings or things. A DTN protocol is concerned with the delivery of messages between nodes within these dynamic networks in a way that is tolerant to intermittent connections, disconnections and failures. Such systems are often characterised by a sparse network of connections between individuals that change over time.

In previous work, it was demonstrated that community structure can be useful for routing in PSNs [6]. The work introduced a particular routing algorithm, BUBBLE Rap, that exploited the structure returned by a particular community finding algorithm. However, it left two questions unanswered: (1) could one improve the results by modifying the routing algorithm, and (2) which community finding algorithm should be used by the routing algorithm? In this paper, we 
present the results of experiments designed to address these questions. The contribution of this paper is the comparison of hierarchical, non-hierarchical, overlapping and non-overlapping community-based message routing using 5 community finding algorithms, on networks derived from multiple real-world datasets. Our BubbleH algorithm is described in more details in Section 3 and in [16].

The paper is organized as follows. In the next section we provide an overview of existing research on community-based message routing. Next, we compare the performance of two routing algorithms, BubbleH and BUBBLE Rap. We find that our BubbleH algorithm performs better than BUBBLE Rap when there is hierarchical community structure. Next, we employ various community finding algorithms and try to identify which is most useful for routing purposes. We try out several types of community finding algorithms - including those which return disjoint, overlapping or hierarchical community information. For all experiments, we use several datasets.

\section{Message Routing}

Research on DTNs has produced many strategies for message routing. Flooding based approaches flood the network with copies of messages between any nodes that meet. Epidemic-like protocols behave similarly, for example Epidemic Routing [17] which transmits messages to other nodes with some probability, but limits messages by hop count to reduce overhead. These are perhaps the most effective, but the large number of message copies generated and sent mean a large overhead in message transmission between nodes. More conservative approaches include Spray and Wait [15], where a limited number of messages are distributed before a phase where nodes keep the messages until meeting the destination. Other approaches involve probabilistic, or opportunistic mechanisms to predict future interactions, such as PROPHET [10] and Context-Aware Adaptive Routing (CAR) [11] which use knowledge about previous contacts to inform predictions about co-locations which are used to decide next-hop routes. Zhang provides a survey of schemes for routing in intermittently connected mobile ad-hoc networks [18], and groups each of the approaches into two distinct categories; deterministic approaches, where the network structure is known over time, and non-deterministic, those where the network structure is not known. In this paper, we are considering deterministic approaches, as the algorithms we are testing assume that the network structure is available to them for community detection. We make the assumption that network structure is available, so that we can concentrate on the underlying operation of the routing algorithm, in a real-world scenario, it is likely that network structure is only known via means of incremental updates as nodes meet each other, this is beyond the scope of this paper, but is of interest for further work.

The effectiveness of a DTN message routing algorithm can be encapsulated in three metrics. Most often the goal is to have high delivery ratio, a low overhead transmission cost and low delivery latency [2]. When comparing the ability of a routing scheme to deliver messages effectively, the relative importance of each metric depends upon the application involved. In the case where a message is being sent between people, for example in a distributed version of the current 
SMS message system, the expectation may be that the delivery ratio is very high and most important. Also important, is that it should have a low latency, as the message should arrive promptly. Less important is that the cost is as low as possible, but not so low that the message never arrives.

In other situations, delivery ratio and latency may not be as important. Where the content of a message is, for example, a personal status broadcast system, perhaps an infrastructure-less, distributed version of Twitter, then delivery ratio may not be as important. We may only want to have the most up to date status updates - old, out of date messages may be discarded, so cost may be our most important metric. For clarity, here we define the above metrics as the following:

Delivery Ratio (average) is the number of delivered messages over the total number of messages sent.

Delivery Latency (average) is the total time to deliver each delivered message. over the total number of delivered messages.

Cost (average) is the total number of transmissions of messages (delivered and undelivered), over the total number of messages sent.

\section{Communities for Routing}

Here we review two routing algorithms that base their routing decisions on community structure: the well-known BUBBLE Rap algorithm [6] and our own BubbleH [16] algorithm, which extends the basic BUBBLE Rap idea to exploit community hierarchy and the comparative size of communities. Any community finding algorithm could be used by these routing algorithms; which we will discuss in the next section.In this way, we hope to be able compare the benefits of each community finding algorithm in terms of each message routing algorithm.

Each of these routing algorithms use the notion of community structure to inform routing decisions. BUBBLE Rap uses Palla et al's Clique Percolation Method [12] (referred to as k-CLIQUE), to generate overlapping community structure from a graph formed from contacts between nodes. BUBBLE Rap uses the notion of local and global node ranking to make decisions about message passing. Node rank is based on the betweenness centrality of each node in the global network (global rank) and each community the node belongs to (local rank). As with the BUBBLE Rap algorithm, the BubbleH algorithm calculates local rankings based on betweenness centrality, however, it does not use global rank. Instead, it uses the community hierarchy to drive the mobility of messages within the network. When a node encounters another, it considers whether to pass the message on based on how close the other node is in the network hierarchy to the destination node. BubbleH has been shown to improve delivery ratio and at least match delivery cost of BUBBLE Rap for the MIT Reality Mining dataset using the H-GCE community finding algorithm[16]. Here we intend to evaluate its performance against multiple datasets and multiple community finding algorithms.

Figure 1 shows the BubbleH algorithm, the Bridging Community $(B C(p, d))$, is the smallest community that contains the node in question, and the recipient, 


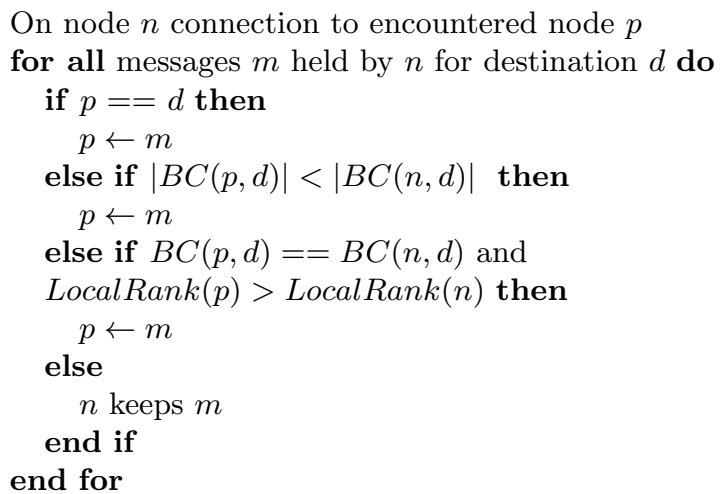

Fig. 1. BubbleH Algorithm, where $B C(p, d)$ is the set of all nodes which represent the smallest community, or Bridging Community containing both node $p$ and node $d$.

or destination node. To find the Bridging Community between two nodes, BubbleH finds all of the communities that the nodes share, and picks the shared community that has the lowest member count. In the case where there are multiple candidate communities (when the community finding algorithm allows overlap), the last candidate community in the list is chosen.

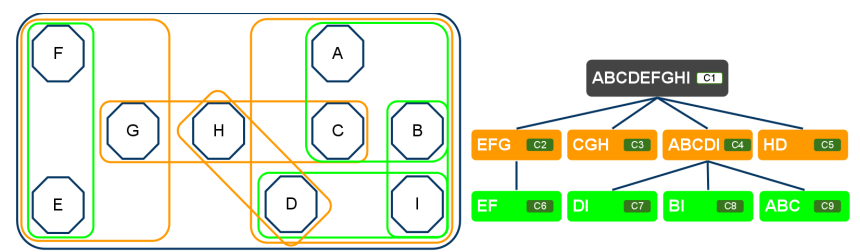

Fig. 2. Simplified example of an overlapping hierarchical community structure. The grouping of nodes in the left section relates to the community hierarchy shown in the right section. Communities are identified as $C 1$ to $C 9$, nodes are identified as $A$ to $G$. For clarity, edges between nodes are not shown.

To illustrate the concept, Figure 2 shows a simplified overlapping community structure, and its associated hierarchical structure. If we imagine that node $F$ has a message destined for node $B$, the smallest community containing both $F$ and $B$ is $c 1$, this is the bridging community for $F$ and $B$. On encountering another node, $F$ must consider whether the encountered node has a better bridging community than itself. For example, when meeting node $C$, whose bridging community with destination node $B$ is $c 9$, it will find that $c 9$ has less members than $c 1$, and pass the message to $C$.

\section{Community Finding}

As described above, any community finding method can be plugged into the routing algorithm. The community finding algorithms that we evaluate here differ fundamentally: whereas InfoMap produces a non-overlapping partition of nodes, the others allow communities to overlap. While Ahn et al.'s link partitioner 
and Hierarchical Greedy Clique Expansion (H-GCE) create a dendrogram of hierarchical structure, the others are based on a flatter conception of community structure. We now provide a brief description of InfoMap, the Clique Percolation Method, the Link Clustering algorithm, and of H-GCE.

InfoMap [13] The basic idea behind InfoMap is that a random walker in a network will tend to get stuck in communities, spending relatively large amounts of time within communities and small amounts of time passing between them. This regularity enables compression of a string representation of the random walk sequence: by assigning a unique namespace to each community, short node IDs can be recycled between communities. The extra cost (in terms of information) for the namespace scheme is that each time the random walker switches namespaces, special exit and entry codes must be inserted into the sequence.

The way the random walker moves around communities can be likened to the flow of messages between individuals, there may be a strong current of messages between well connected individuals within communities, and less so to other communities.

Rosvall and Bergstrom show that given a partition, one can efficiently calculate how much compression can be obtained due to community structure with the "map equation." The map equation can then be maximized by any search algorithm. For an explanation of InfoMap, see www.mapequation.org.

k-CLIQUE (Clique Percolation Method) [12] In 2005 Palla et al. introduced their clique percolation method for community finding called CFinder. Clique percolation entails finding $k$-clique communities which are the union of cliques of size $k$ respecting the constraint that these cliques are adjacent in the sense that they share $k-1$ nodes.

In their paper describing BUBBLE Rap [6], Hui et al use this method for finding communities, which they refer to as the k-CLIQUE method. Hui et. al. remove edges of the original node graph before clustering based on a threshold of connected time for each edge. We also implement this threshold version and tune the threshold weight for each dataset as described in section 4.1.

Link Clustering [1] With their work on link partitioning Ahn et al. take an edge-centric view of communities, defining a community as a set of edges rather than a set of nodes. Their aim is to partition all edges in the graph into nonoverlapping communities. Although under this scheme each edge must belong to exactly one community, nodes can belong to multiple communities. The objective function they propose is simply the normalized edge density of each community, where each community is weighted by the fraction of edges present in it. In their algorithm they use single-linkage hierarchical clustering to create a dendrogram, and cut the dendrogram where the objective function is maximized. We adapted the reference implementation of LinkClustering ${ }^{1}$ to discard edges under a given threshold, in the same way as with k-CLIQUE.

Random The random community finding algorithm assigns nodes to communities at random, ensuring that communities do not overlap by more than $50 \%$.

\footnotetext{
${ }^{1}$ http://barabasilab.neu.edu/projects/linkcommunities/
} 
H-GCE Agglomerative community finding algorithms start with a set of seeds (where a seed is typically a node or an edge), and expands these seeds by adding nodes to them such that a local fitness function is greedily optimized. In [9], Lee et al. showed that cliques provide good seeds for these algorithms, especially in networks where nodes belong to several communities.

In [16] we proposed H-GCE, an agglomerative community finding algorithm that uses cliques as seeds and additionally uses random perturbations to identify which communities are stable. The idea is that "significant" communities will be recoverable even if some noise is added to the graph; this idea has been previously developed in $[7,14]$. This and other approaches to measuring the significance of communities are outlined in Fortunato's review of community finding methods [4]. For the details of H-GCE, see [16].

\subsection{Community Finding Data}

To build a network for the community finding analysis we add an undirected edge between individual if they met during the simulation time period and we weight the edge by total amount of time the nodes were connected to each other. Each algorithm deals with edge weights differently; for KCLIQUE, LinkClustering and HGCE, we specify a threshold cut value for the network edges. InfoMap and Random do not take a threshold parameter.

Threshold Selection We use the the mean, median, 80th percentile and 20th percentile of the connected time for all edges as threshold values. This gives us a robust way to get a good approximation for threshold values. However, this may not be the optimum mechanism for calculating threshold values as it is based on our experience in early experiments. In our results, we show the best results for each metric (delivery ratio, latency, cost etc.), for each routing algorithm to give them a fair chance against each other.

\section{$5 \quad$ Message routing using BubbleH}

In our previous work [16], we showed that BubbleH performs well against BUBBLE Rap when using a hierarchical community finding algorithm. In the experiment described here, we use the same Bluetooth proximity traces from the MIT Reality project [3] (described in section 6) to drive contact events within the simulator. The MIT Reality dataset has the most number of connections between between Oct 2004 and Jan 2005, so we chose the period between Nov 2004 and Dec 2004 for community detection and testing, which we refer to as MIT-NOV.

Figure 3 shows a visualisation of the MIT-NOV dataset, where edges are weighted by connected time, and nodes are clustered using the InfoMap algorithm (with clusters indicated by node colour).

\subsection{BubbleH versus BubbleRAP evaluation}

This simulation tests message routes between all pairs of nodes in the dataset. In the evaluation we consider three different criteria, the relative importance of these criteria may depend on specific circumstances but a reasonable assessment of the order of importance is as follows: 


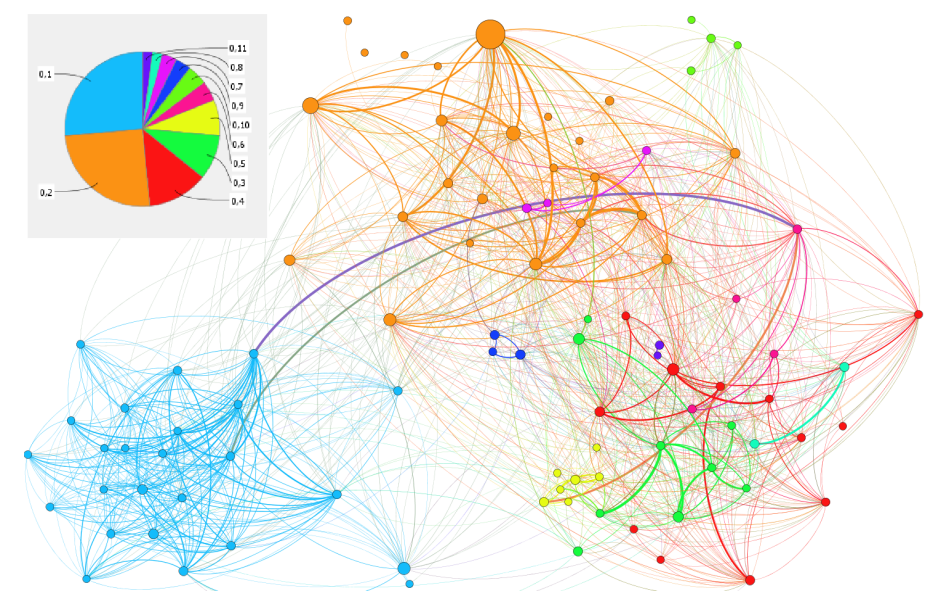

Fig. 3. Visualisation of the MIT-NOV dataset, node and edge colour shows the assigned cluster using the InfoMap algorithm. Node size represents betweenness centrality. Edge thickness represents total connected time between nodes. Also shown inset, is the proportion of nodes included in each community. For clarity, this graph does not show edges where the total connected time is less than the median for all edges.

1. Delivery Ratio: The proportion of messages successfully delivered. Delivery Ratio $=$ total number of delivered messages / total number of messages sent

2. Latency: This measures the amount of time it takes to deliver messages. Latency $=$ total time to deliver messages / total delivered Messages

3. Cost: This counts the average number of 'hops' it takes to deliver messages. Cost $=$ total number of hops to deliver messages / total delivered messages

Since Latency and Cost are measured only over delivered messages it is important to know the proportion of messages that are successfully delivered. At the same time it is important to remember that the simulation attempts to pass messages between all pairs of nodes so some of these source/destination pairs would never arise in practice.

The importance of Cost as an evaluation criterion is difficult to assess without a specific application context. In some scenarios it may not matter whether a message passes through three or six nodes on its way to the destination. On the other hand a flooding strategy does not appear to be reasonable either.

\subsection{Simulation}

To evaluate the performance of BubbleH, we have used ContactSim a discrete time event simulator designed specifically for evaluating DTN routing. ContactSim is capable of using contact traces recorded either during real-world experiments or generated synthetically.

Results In figure 4 we show the results of routing based on the communities found using four community finding algorithms applied to MIT-NOV. In addition to KCLIQUE and HGCE, we tested InfoMap and LinkClustering to see if 

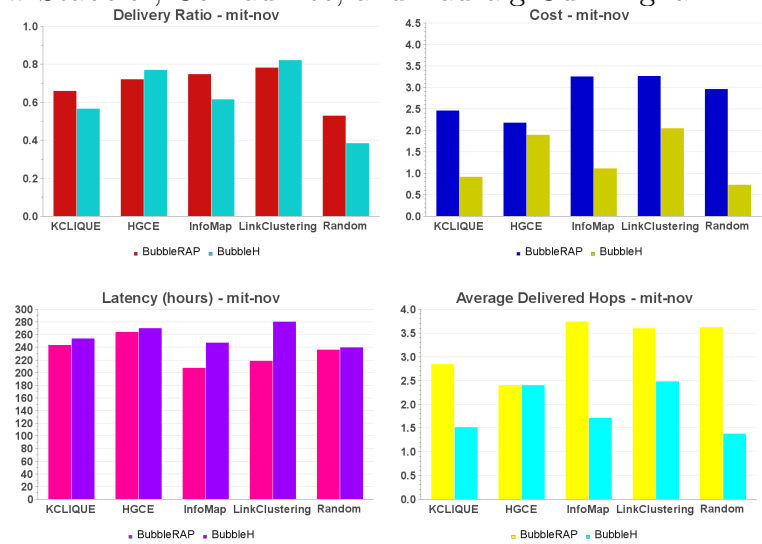

Fig. 4. Result of routing using BubbleH and BubbleRAP on the MIT-NOV dataset, showing the delivery ratio, cost, latency and the number of delivered hops.

the overlapping and hierarchical nature of LinkClustering would achieve better results that the non-overlapping InfoMap.

In this case, we see that BubbleH performs well in terms of delivery ratio when using the hierarchical and overlapping community finding algorithms HGCE and LinkClustering. It also does well in terms of cost for all methods. Latency is impacted overall (perhaps because some more difficult messages are being delivered), but the number of hops taken to deliver the messages is lower in most cases, suggesting that BubbleH is more selective than BUBBLE Rap, and 'holds' messages for longer, to ensure a more direct route.

\section{Evaluation}

In the first experiment described above, we sent messages between all pairs of nodes, over the entire dataset period. However, we also wanted to test how well our algorithms coped using a more realistic approach. To achieve this, we split the datasets into two distinct periods; a training period, in which the community finding algorithms are allowed to find communities; and a test period, in which the routing algorithm tries to deliver messages. We only send messages between nodes that communicated during the training phase, meaning only selected pairs of nodes were used. We believe this to be a more realistic experimental set-up, as in practice we would not expect all nodes to communicate with every other node. We expect this to result in a higher delivery ratio.

We chose five datasets with which to test our algorithms, here we briefly describe each dataset we use for our simulations. Two of the datasets (Enron and Studivz) don't represent real DTN scenarios but do have the contact characteristics required for the simulation.

MIT Reality The authors have captured a trace of human contacts over a period of time: communication, proximity, location, and activity information from 100 subjects at MIT over the course of the 2004-2005 academic year. We chose a 4 month section in the most active period (Nov 2004 to Feb 2005); the first month for training, and the remaining three months as the test period. We refer to this as MIT-SPLIT. 
Enron Email Corpus This dataset, introduced by Klimt and Yang [8], comprises a large set of emails made public during the legal investigation concerning the Enron corporation. We used the occurrence of an email sent between individuals to represent a physical contact with a duration of 1 second. In this dataset we picked a busy three month period, between April 2001 and July 2001 we refer to this dataset as ENRON-SPLIT-A.

Social Sensing The Social Sensing Study was undertaken by the CLARITY centre for sensor web technologies and took place at University College Dublin and Dublin City University. Mobile phones carried by participants collected data about their interactions, including nearby Bluetooth devices, cell towers and WiFi Access points. We chose a 6 month period for our simulation between Jan 2009 and June 2009, we designated the first two months as the training period. We refer to this dataset as SOCIAL-SENSING-SPLIT.

Studivz This dataset is derived from wall posts from a university social networking site Studivz ${ }^{2}$, we determine contacts occur when a post is made on the 'wall' of another person. Each contact event is given a duration of 1 second per character in the wall post. We chose an active period of three months (from Oct 2007) to use, resulting in a network containing over 28 thousand nodes. To reduce the dataset to a more manageable size, comparable with other datasets we evaluate, we randomly selected three nodes from the aggregate graph of the three month period, and followed the edges to a depth of 2 hops, this resulted in sub-graph of 385 nodes. We refer to this as STUDIVZ-SPLIT

Hypertext2009 This dataset was collected during the ACM Hypertext 2009 conference, where the SocioPatterns ${ }^{3}$ project deployed the Live Social Semantics application. Conference attendees volunteered to wear radio badges that monitored their face-to-face proximity. The dataset represents the dynamical network of face-to-face proximity of 110 conference attendees over about 2.5 days. We used the first day for training and the remaining time for the test period, we refer to this as HYPERTEXT2009-SPLIT.

\subsection{Results}

Figure 5 shows the resulting delivery ratio for BUBBLE Rap and BubbleH, when routing over five datasets, for each community finding algorithm. We notice that for MIT-SPLIT, there is not such a pronounced improvement by BubbleH. The story is the same for the other datasets, where there is little difference in delivery ratio between BUBBLE Rap and BubbleH, however interestingly, we see that in most cases, InfoMap and LinkClustering perform better than KCLIQUE, HGCE and Random. The flow based approach of InfoMap and the large number of overlapping community structures of LinkClustering appear to make for better routing decisions.

In terms of transmission cost, shown in Figure 6, we see that BubbleH generally performs better than BUBBLE Rap, H-GCE makes distinct improvements in cost for BubbleH in the MIT-SPLIT and HYPERTEXT2009-SPLIT datasets.

\footnotetext{
${ }^{2}$ http://www. studivz.net/

3 http://www.sociopatterns.org
} 


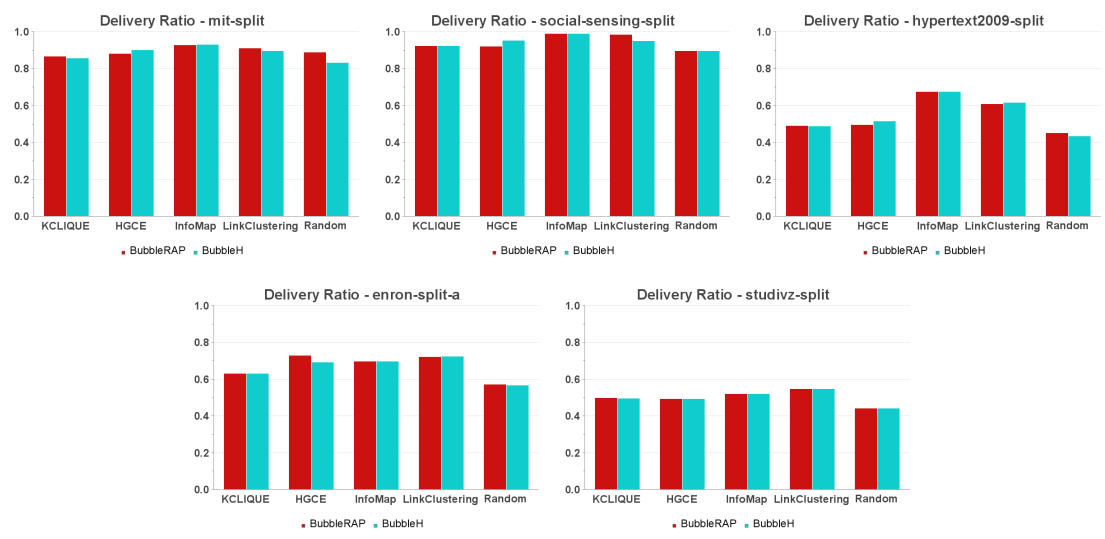

Fig. 5. Delivery Ratio for BUBBLE Rap and BubbleH, for five datasets.

The results in terms of latency, in Figure 7 are not as clear, in MIT-SPLIT, InfoMap and LinkClustering perform well, but BUBBLE Rap and BubbleH have nearly an equal number of wins. The STUDIVZ-SPLIT dataset shows a very poor latency, and a very low cost. This suggests that the network is very sparsely connected, perhaps with many of the nodes only contacting each other very occasionally.

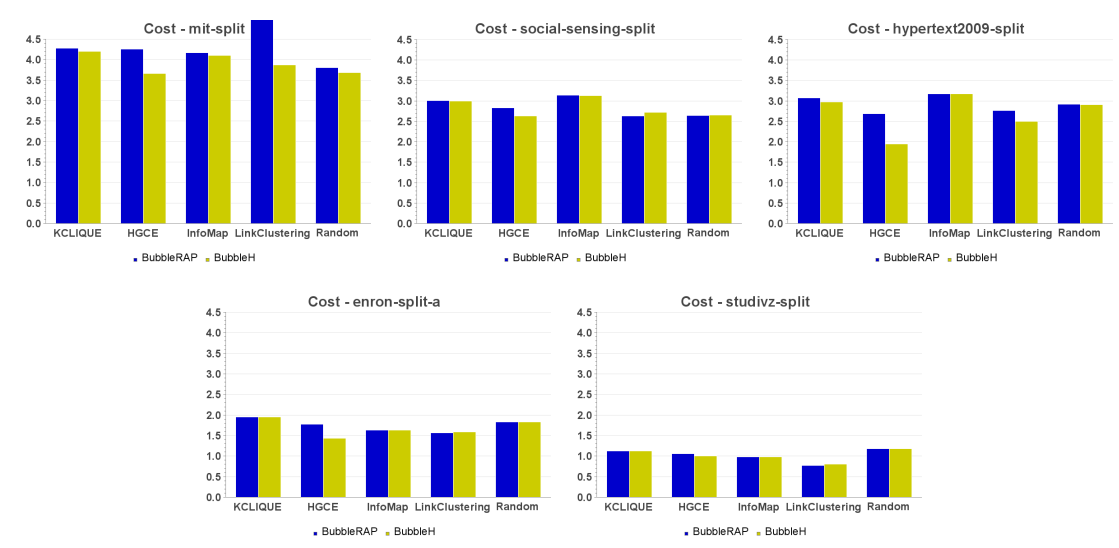

Fig. 6. Cost for BUBBLE Rap and BubbleH, for five datasets.

It is interesting that $\mathrm{H}-\mathrm{GCE}$ performs well in the real-world contact datasets, suggesting that there may be a distinct difference between real-world contacts, and virtual contacts. There is a clear difference in results between the MIT-NOV from the first experiment and the MIT-SPLIT dataset, this is due to the nature of how the message pairs were selected. In the case of the SPLIT datasets, only pairs who communicated during the training phase created messages to be delivered to one another. 


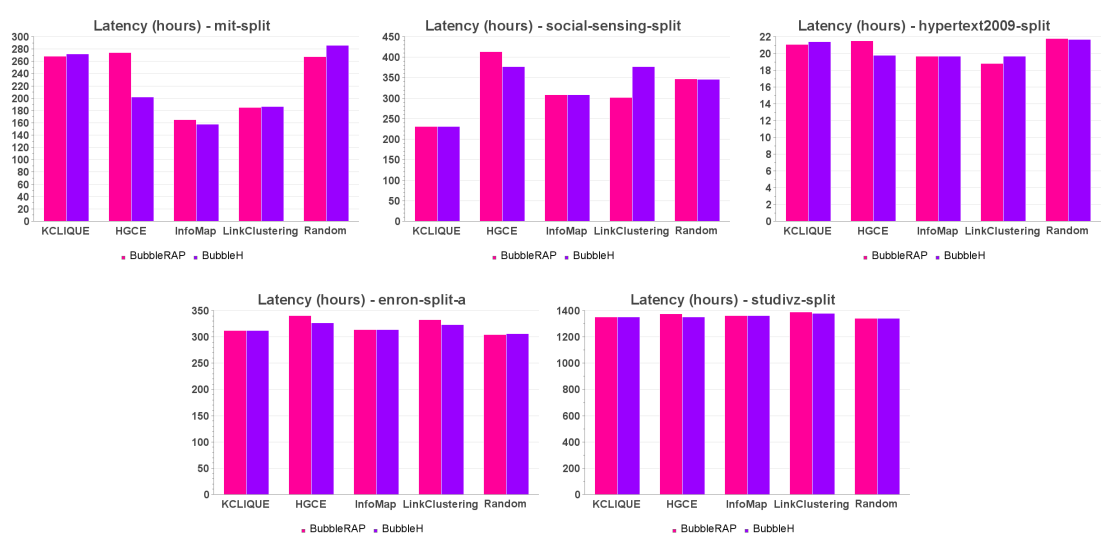

Fig. 7. Latency for BUBBLE Rap and BubbleH, for five datasets.

\section{Conclusion and Future Work}

In this paper, we show that our hierarchicy based community messaging algorithm BubbleH, performs better than it's non-hierarchical predecessor, BUBBLE Rap, on five different datasets. We have also evaluated a number of community finding strategies, and have found that LinkClustering with its overlapping hierarchical clustering, and InfoMap, with it's flat partitioning, perform well. InfoMap's success may be due to the optimization that drives it; based on the concept of flow between communities. Perhaps the combination of LinkClustering's highly overlapping and hierarchical approach gives it the edge over the other algorithms.

Future work should explore the extent to which community finding affects the results of routing schemes on different types of dataset, in this way, we may be able to benchmark different community finding approaches, by means of DTN routing performance tests.

A further challenge is the need to incorporate a scheme that includes the dissemination of network structure data in an efficient manner, which will allow nodes to individually calculate community structures, allowing the system to be truly distributed.

Another aspect that has not been fully explored in the literature, is how to use a node's physical location as a driver for routing in communities. Future work should examine new ways to use a persons location to firstly, affect community finding, and secondly, aid routing.

\section{Acknowledgements}

This work is supported by a PhD scholarship from the Irish Research Council for Science Engineering and Technology and by Science Foundation Ireland Grant No. 08/SRC/I140 (Clique: Graph and Network Analysis Cluster).

\section{References}

1. Ahn, Y., Bagrow, J., Lehmann, S.: Link communities reveal multiscale complexity in networks. Nature 466(7307), 761-764 (2010) 
2. Crowcroft, J., Yoneki, E., Hui, P., Henderson, T.: Promoting tolerance for delay tolerant network research. SIGCOMM Comput. Commun. Rev. 38(5), 63-68 (2008)

3. Eagle, N., (sandy) Pentland, A.: Reality mining: sensing complex social systems. Personal and Ubiquitous Computing 10(4), 255-268 (May 2006)

4. Fortunato, S.: Community detection in graphs. Physics Reports 486(3-5), 75-174 (2010)

5. Hui, P., Chaintreau, A., Scott, J., Gass, R., Crowcroft, J., Diot, C.: Pocket switched networks and human mobility in conference environments. Proceeding of the 2005 ACM SIGCOMM workshop on Delay-tolerant networking - WDTN '05 pp. 244-251 (2005)

6. Hui, P., Crowcroft, J., Yoneki, E.: Bubble rap: social-based forwarding in delay tolerant networks. In: Proceedings of the 9th ACM international symposium on Mobile ad hoc networking and computing. pp. 241-250. MobiHoc '08, ACM, New York, NY, USA (2008)

7. Karrer, B., Levina, E., Newman, M.E.J.: Robustness of community structure in networks. Physical Review E 77(4), 046119 (Apr 2008)

8. Klimt, B., Yang, Y.: Introducing the Enron corpus. In: First conference on email and anti-spam (CEAS) (2004)

9. Lee, C., Reid, F., McDaid, A., Hurley, N.: Seeding for pervasively overlapping communities. Phys. Rev. E 83, 066107 (Jun 2011)

10. Lindgren, A., Doria, A., Schelén, O.: Probabilistic routing in intermittently connected networks. ACM SIGMOBILE Mobile Computing and Communications Review 7(3), 19 (Jul 2003)

11. Musolesi, M., Mascolo, C.: CAR: Context-Aware Adaptive Routing for DelayTolerant Mobile Networks. IEEE Transactions on Mobile Computing 8(2), 246-260 (Feb 2009)

12. Palla, G., Derényi, I., Farkas, I., Vicsek, T.: Uncovering the overlapping community structure of complex networks in nature and society. Nature 435(7043), 814-818 (2005)

13. Rosvall, M., Axelsson, D., Bergstrom, C.: The map equation. The European Physical Journal-Special Topics 178(1), 13-23 (2009)

14. Rosvall, M., Bergstrom, C.: Mapping change in large networks. arXiv pp. 1-9 (2008)

15. Spyropoulos, T., Psounis, K., Raghavendra, C.S.: Spray and wait: an efficient routing scheme for intermittently connected mobile networks. In: Proceedings of the 2005 ACM SIGCOMM workshop on Delay-tolerant networking. pp. 252-259. WDTN '05, ACM, New York, NY, USA (2005)

16. Stabeler, M., Lee, C., Williamson, G., Cunningham, P.: Using Hierarchical Community Structure to Improve Community-Based Message Routing. In: ICWSM-11 Workshop on Social Mobile Web Workshop (SMW-11) (2011)

17. Vahdat, A., Becker, D.: Epidemic Routing for Partially Connected Ad Hoc Networks (2000)

18. Zhang, Z.: Routing in intermittently connected mobile ad hoc networks and delay tolerant networks: overview and challenges. IEEE Communications Surveys \& Tutorials 8(1), 24-37 (2006) 